\title{
The complete hyper-surfaces with zero scalar curvature in $\mathbb{R}^{n+1}$
}

\author{
Li Yaowen · Xu Xingwang · Zhou Jiuru
}

Received: 11 January 2013 / Accepted: 24 March 2013 / Published online: 8 April 2013

(C) The Author(s) 2013. This article is published with open access at Springerlink.com

\begin{abstract}
Let $M^{n}$ be a complete and noncompact hyper-surface immersed in $R^{n+1}$. We should show that if $M$ is of finite total curvature and Ricci flat, then $M$ turns out to be a hyperplane. Meanwhile, the hyper-surfaces with the vanishing scalar curvature is also considered in this paper. It can be shown that if the total curvature is sufficiently small, then by refined Kato's inequality, conformal flatness and flatness are equivalent in some sense. And those results should be compared with Hartman and Nirenberg's similar results with flat curvature assumption.
\end{abstract}

Keywords Bernstein type theorems $\cdot$ Scalar curvature $\cdot$ Sobolev inequality $\cdot$ Ends

Mathematics Subject Classification (2000) Primary 53C20 ;

Secondary 53A07 $\cdot 53 \mathrm{~A} 10$

\section{Introduction}

The classical Bernstein theorem states that a minimal graph $M^{n}(n \leq 7)$ immersed in $\mathbb{R}^{n+1}$ must be a hyperplane $[3,8,19]$. Basically, this beautiful theorem claims that certain elliptic non-linear differential equation on the whole space only has standard solutions. Such result is in the fashion of Louiville theorem for bounded harmonic functions on the whole space. Since then, this result has been generalized to the various hyper-surfaces such as the stable

\footnotetext{
L. Yaowen

Department of Mathematics, Nanjing University, Nanjing 210093, Peoples Republic of China e-mail: lieyauvn@263.net
}

X. Xingwang $\cdot$ Z. Jiuru $(\bowtie)$

Department of Mathematics, National University of Singapore, 2 Science Drive 2,

Singapore 117543, Republic of Singapore

e-mail: zhoujr.1982@yahoo.com.cn

X. Xingwang

e-mail: matxuxw@nus.edu.sg 
and constant mean curvature hyper-surfaces by many authors [4,5], etc., and the parametric minimal hyper-surfaces with finite total curvature by other group of mathematicians $[7,18$, 20]. Notice that the mean curvature is just the trace of the second fundamental form. Hence, it will be equally interesting to consider other elementary symmetric functions of the second fundamental form. In particular, it is natural to ask whether hyper-surfaces with zero scalar curvature have Bernstein type property $[2,6,10,17]$. However, observe that the equation for the hyper-surfaces with zero mean curvature is elliptic, the analogy for surfaces with zero scalar curvature is only a degenerate elliptic equation. Thus for hyper-surfaces with the zero scalar curvature, we cannot expect the results being as nice as the ones for minimal hypersurfaces. On the other hand, we notice that there is another classical result given by Hartman and Nirenberg, which says that a complete hyper-surface with zero sectional curvature is either a hyperplane or a generalized cylinder. After we got some partial results for hypersurfaces with zero scalar curvature, the simple question we may ask is what happens if $M$ is Ricci flat. This article will report what we have got so far along this direction.

First let us fix some standard notation. Let $M^{n}$ be a complete and noncompact hypersurface isometrically immersed in $\mathbb{R}^{n+1}$. We denote the normalized mean curvature by $\mathrm{H}$, the second fundamental form by $B$ or $\left\{h_{i j}\right\}$ under a local coordinate system. For convenience, we call $\left(\int_{M}|H|^{n} \mathrm{~d} v\right)^{\frac{1}{n}}$ the total curvature of $M$. In the following, we will always assume $M$ is orientable with a fixed orientation. Our first result is the following Bernstein type theorem:

Theorem 1.1 Let $M^{n}(n>2)$ be a complete and noncompact hyper-surface immersed in $\mathbb{R}^{n+1}$ with zero Ricci curvature. If the total curvature is finite, then $M$ is a hyperplane.

Remark One should compare Theorem 1.2 with the result of Hartman and Nirenberg [9]. Notice that even $M$ is of zero sectional curvature and $M$ may not be a hyperplane. A typical example is the so-called generalized cylinder given by $\left(x_{1}, x_{2}, \ldots, x_{n}\right) \in \mathbb{R}^{n} \longrightarrow$ $\left(x_{1}, x_{2}, \ldots, x_{n}, \cosh \left(x_{n}\right)\right) \in \mathbb{R}^{n+1}$. Thus our second condition is to get rid of the generalized cylinders.

Next we consider the hyper-surfaces with the vanishing scalar curvature. Observe that there are many examples of zero scalar curvature hyper-surfaces with finite total curvature which are not flat. See the examples provided by Lounie and Leite [13]. Clearly the analogy of our previous theorem with only the scalar flat assumption cannot be true. Nevertheless, we obtain the following result:

Theorem 1.2 Let $M^{n}(n \geq 3)$ be a complete sub-manifold immersed in $R^{n+1}$ with zero scalar curvature. There exists a sufficiently small number $\alpha$ which depends only on dimension $n$ such that if

$$
\left(\int_{M}|H|^{n} \mathrm{~d} v\right)^{\frac{1}{n}}<\alpha,
$$

then the following statement are equivalent:

(a) $M$ is locally conformally flat;

(b) $|\nabla B|^{2}=n^{2}|\nabla H|^{2}$;

(c) $n H \cdot \operatorname{tr}\left(B^{3}\right)=n^{4} H^{4}$;

(d) $M$ is flat.

Notice that the curvature tensor can be decomposed into the Weyl tensor, Ricci tensor and scalar part. The flatness assumption means all Weyl, Ricci and scalar part vanish and Ricci 
flatness just simply means both Ricci and scalar part vanish. Clearly, the vanishing condition on sole one of three tensors in this decomposition is not enough to conclude the flatness. Thus it seems our above assumptions are reasonable to conclude the flatness. On the other hand, the flat hyper-surface has only one end while the circled cylinder $\left(S^{1} \times \mathbb{R}^{n-1}\right)$ has two ends. In fact, with just concern of the number of ends, we have the following:

Theorem 1.3 Let $M^{n}(n \geq 3)$ be a complete sub-manifold immersed in $R^{n+1}$ with zero scalar curvature. There exists a positive constant $C_{2}>0$ such that if

$$
\left(\int_{M}|H|^{n} \mathrm{~d} v\right)^{\frac{1}{n}}<C_{2},
$$

then M has only one end.

The organizing of paper is as follows: in section two, we will list several useful Lemmas which will be used in the rest of paper. In section three, we will give a proof of our first result, i.e. Theorem 1.1. We should point out that what we really proved in this section is that if $M$ is Ricci flat, then $M$ is flat. Hence a famous result of Hartman and Nirenberg implies that $M$ is either hyperplanes or generalized cylinders. And in section four, we prove our second result, i.e. Theorem 1.2. The main observation here is to fully use the assumption that the scalar curvature is zero. This condition implies a differential identity for mean curvature and the second fundamental form. Together with our assumption, this identity implies that $M$ is flat hyper-surface and hence the conclusion follows as before. In the final section, we make several comments on number of ends of the hyper-surfaces under various assumptions and prove Theorem 1.3. This is motivated by similar result for either minimal hyper-surfaces or constant mean curvature hyper-surfaces. The key assumption is that the dimension is of at least three.

\section{Several useful lemmas}

First the main fact we should use for zero scalar curvature hyper-surfaces is the following Lemma:

Lemma 2.1 Let $M^{n}$ be a hyper-surface immersed in $\mathbb{R}^{n+1}$ with scalar curvature $R$. Then we have

$$
R=n^{2} H^{2}-|B|^{2} .
$$

Proof This is well known and can be found in any Riemannian geometry book.

Based on this identity, we have

Lemma 2.2 Let $M^{n}$ be a hyper-surface isometrically immersed in $R^{n+1}$ with constant scalar curvature $R$. Then the following identity holds true:

$$
n\left(n H \delta_{i j}-h_{i j}\right) H_{i j}=|\nabla B|^{2}-n^{2}|\nabla H|^{2}+n H \operatorname{tr}\left(B^{3}\right)-\left(n^{2} H^{2}-R\right)^{2} .
$$

Thus if $R \equiv 0$, then

$$
|\nabla B|^{2} \geq n^{2}|\nabla H|^{2}
$$

as shown in [1]. 
Proof Recall that for such hyper-surfaces, the curvature tensor is given by

$$
R_{i j k l}=h_{i k} h_{j l}-h_{i l} h_{j k} .
$$

Hence differentiate the identity (2.1) to get

$$
\sum_{i, j=1}^{n} h_{i j} h_{i j, k}=n^{2} H H_{k}
$$

And differentiate once again in the direction $e_{k}$ and sum them to get

$$
|\nabla B|^{2}+\sum_{i, j, k=1}^{n} h_{i j} h_{i k, j k}=n^{2}|\nabla H|^{2}+n^{2} H \Delta H .
$$

Here we have used the fact that $h_{i j, k}=h_{i k, j}$ for all $i, j, k$. Now Ricci identity and Lemma 2.1 give

$$
\begin{aligned}
\sum_{k=1}^{n} h_{i k, j k} & =\sum_{k=1}^{n} h_{i k, k j}+\sum_{k, l=1}^{n} h_{l k}\left(h_{l j} h_{i k}-h_{l k} h_{i j}\right)+\sum_{k, l=1}^{n} h_{i l}\left(h_{l j} n H-h_{l k} h_{j k}\right) \\
& =n H_{, i j}+n H \sum_{l=1}^{n} h_{i l} h_{l j}-\left(n^{2} H^{2}-R\right) h_{i j} .
\end{aligned}
$$

Multiply $h_{i j}$ to both sides of above identity and sum up for $i, j$ from 1 to $n$ and rearrange the terms to get the identity (2.2).

To get the last inequality, we square both sides of the Eq. (2.3) and sum up with respect to $k$ to get

$$
n^{4} H^{2}|\nabla H|^{2}=\sum_{k=1}^{n}\left(\sum_{i, j=1}^{n} h_{i j} h_{i j, k}\right)^{2} .
$$

Notice that Cauchy-Schwartz inequality shows that

$$
\begin{aligned}
\sum_{k=1}^{n}\left(\sum_{i, j=1}^{n} h_{i j} h_{i j, k}\right)^{2} & \leq \sum_{k=1}^{n}\left(\sum_{i j} h_{i j}^{2}\right)\left(\sum_{i j} h_{i j, k}^{2}\right) \\
& =n^{2} H^{2}|\nabla B|^{2} .
\end{aligned}
$$

Hence if $H \neq 0$, then $|\nabla B|^{2} \geq n^{2}|\nabla H|^{2}$. If $H=0$ at some point, then $h_{i j}=0$ at that point. Hence the Eq. (2.2) shows that $|\nabla B|^{2}=n^{2}|\nabla H|^{2}$ at that point. Therefore, the desired inequality holds at all points.

Lemma 2.3 Let $a_{i}, i=1,2, \ldots, n$, be real numbers satisfying

$$
\sum_{i=1}^{n} a_{i}=0, \sum_{i=1}^{n} a_{i}^{2}=|\mu|^{2}
$$

Then we have

$$
\left|\sum_{i=1}^{n} a_{i}^{3}\right| \leq \frac{n-2}{\sqrt{n(n-1)}}|\mu|^{3} .
$$

The equality holds if and only if $n-1$ terms of $\left\{a_{i}\right\}_{i=1}^{n}$ are equal. 
Proof This is also well known. It follows from Lagrange multiplier method. For the detail, we refer readers to [15].

Lemma 2.4 Let $M^{n}$ be a sub-manifold immersed in $\mathbb{R}^{n+p}$. Then for any function $h \in C_{0}^{1}(M)$, we have

$$
\left(\int_{M}|h|^{\frac{n}{n-1}} \mathrm{~d} M\right)^{\frac{n-1}{n}} \leq C_{1}\left(\int_{M}|\nabla h| \mathrm{d} M+n \int_{M}|H h| \mathrm{d} M\right) .
$$

Proof This is proved by Michael and Simon [14] or Hoffman and Spruck [11], respectively.

We only need its following corollary:

Lemma 2.5 Let $M^{n}$ be a sub-manifold immersed in $\mathbb{R}^{n+p}$. Suppose that $n\|H\|_{n} C_{1}<1$ where $C_{1}$ is a constant in Lemma 2.4. Then for any $f \in C_{0}^{1}(M)$, we have

$$
\left(\int_{M}|f|^{\frac{2 n}{n-2}} \mathrm{~d} M\right)^{\frac{n-2}{n}} \leq C_{S} \int_{M}|\nabla f|^{2} \mathrm{~d} M,
$$

where $C_{s}=\left(\frac{C_{1}}{1-n\|H\|_{n} C_{1}} \frac{2(n-1)}{n-2}\right)^{2}$.

Proof For a function $h$ as in Lemma 2.4, by Hölder inequality, one has

$$
\int_{M}|h H| \mathrm{d} M \leq\left(\int_{M} h^{\frac{n}{n-1}} \mathrm{~d} M\right)^{\frac{n-1}{n}}\|H\|_{n} .
$$

Hence, this inequality and Lemma 2.4 imply

$$
\left(\int_{M} h^{\frac{n}{n-1}} \mathrm{~d} M\right)^{\frac{n-1}{n}} \leq \frac{C_{1}}{1-n\|H\|_{n} C_{1}} \int_{M}|\nabla h| \mathrm{d} M .
$$

Now for any $\varphi \in C_{0}^{1}$, we set $h=\varphi^{\frac{2(n-1)}{n-2}}$. Thus we get:

$$
\begin{aligned}
\left(\int_{M} \varphi^{\frac{2 n}{n-2}} \mathrm{~d} M\right)^{\frac{n-1}{n}} & \leq \frac{C_{1}}{1-n\|H\|_{n} C_{1}} \frac{2(n-1)}{n-2} \int_{M}\left|\varphi^{\frac{n}{n-2}} \nabla \varphi\right| \mathrm{d} M \\
& \leq \frac{C_{1}}{1-n\|H\|_{n} C_{1}} \frac{2(n-1)}{n-2}\left(\int_{M}|\varphi|^{\frac{2 n}{n-2}}\right)^{\frac{1}{2}}\left(\int_{M}|\nabla \varphi|^{2} \mathrm{~d} M\right)^{\frac{1}{2}} .
\end{aligned}
$$

Thus the lemma is proved.

The next lemma is to get the volume control from below which will be useful in the course of proof of our main results:

Lemma 2.6 Let $M^{n}(n \geq 3)$ be a complete noncompact immersed hyper-surface in $\mathbb{R}^{n+1}$. Assume that $n C_{1}\|H\|_{n}<1$ where $C_{1}$ is again the constant given in Lemma 2.4. Then there exists a constant $C_{2}>0$ depending only upon $C_{1}$ so that

$$
\operatorname{Vol}(B(q, s)) \geq s^{n\left(1-n C_{1}\|H\|_{n}\right)},
$$


for any $q \in M$, and all $s \geq 0$.

Proof Take an arbitrary point $p \in M$; without loss of generality, we may assume $p=0$. In the following, we let $d(\cdot, \cdot)$ be the distance function of $\mathbb{R}^{n+1}$, and $r(\cdot, \cdot)$ the distance function of $M$ with respect to the induced metric. We will write $d(x), r(x)$ if the base point is 0 . Obviously $d \leq r$ for any two points in $M$. Let $\gamma$ be a minimal geodesic from 0 ; then,

$$
\begin{aligned}
\frac{\partial d}{\partial r} & =\lim _{t \rightarrow 0} \frac{d(\gamma(s+t))-d(\gamma(s))}{t} \\
& \leq \lim _{t \rightarrow 0} \frac{d(\gamma(s+t), \gamma(s))}{t} \quad \text { (by the triangle inequality) } \\
& \leq 1 . \quad(\text { since } d \leq r) .
\end{aligned}
$$

By a direct computation, one can show that

$$
\Delta_{M} d^{2}(x)=2 n(1+H\langle\eta, x\rangle),
$$

where $\eta$ is out unit normal to the hyper-surface $M$ and $x$ is the position vector in $\mathbb{R}^{n+1}$. In particular, $|\langle\eta, x\rangle| \leq d(x) \leq r(x)$.

Let $B(s)$ be the geodesic ball of $M$, of radius $s$ centered at 0 . Integrating the above equation over $B(s)$ and using (2.8) and Hölder inequality, we obtain

$$
2 n \operatorname{vol}(B(s)) \leq 2 s \operatorname{vol}(\partial B(s))+2 n\left(\int_{B(s)}|H|^{n} \mathrm{~d} \mu\right)^{\frac{1}{n}} s(\operatorname{vol}(B(s)))^{\frac{n-1}{n}} .
$$

Since the Sobolev inequality (2.6) holds on $M$, we have the iso-perimetric inequality, namely

$$
\frac{1-n\|H\|_{n} C_{1}}{C_{1}}(\operatorname{vol}(B(s)))^{\frac{n-1}{n}} \leq \operatorname{vol}(\partial B(s)) .
$$

And also note that in any manifold,

$$
\operatorname{vol}(\partial B(s))=\left.\frac{\partial}{\partial r} \operatorname{vol}(B(r))\right|_{r=s} .
$$

We thus obtain

$$
\left.\frac{\partial}{\partial r}\right|_{r=s} \ln (\operatorname{vol}(B(r)))-\frac{n\left(1-n C_{1}\|H\|_{n}\right)}{s\left(1-n C_{1}\left(\|H\|_{n}-\left(\int_{B(s)}|H|^{n} \mathrm{~d} \mu\right)^{\frac{1}{n}}\right)\right)} \geq 0 .
$$

Therefore, by integrating it over the interval $(0, s)$ and taking the exponential to get

$$
\operatorname{vol}(B(s)) \geq s^{n\left(1-n C_{1}\|H\|_{n}\right)},
$$

by observing that $\|H\|_{n}-\left(\int_{B(s)}|H|^{n} \mathrm{~d} \mu\right)^{\frac{1}{n}} \geq 0$.

\section{Proof Of Theorem 1.1}

The proof of Theorem 1.1 is relatively easy. Observe that if Ricci curvature is zero, then we have

$$
h_{i j} h_{j k}=n H h_{i k}
$$


Therefore, we have

$$
\operatorname{Tr}\left(B^{3}\right)=h_{i j} h_{j k} h_{i k}=n H \sum_{i, k=1}^{n}\left(h_{i k}\right)^{2} .
$$

Thus by Lemma 2.1 , since $R=0$, we got

$$
\operatorname{Tr}\left(B^{3}\right)=n^{3} H^{3} .
$$

Now one sets $\mu_{i j}:=h_{i j}-H \delta_{i j}$. Then by simple calculation, we have

$$
|\mu|^{2}=(n-1) n H^{2}
$$

and

$$
\begin{aligned}
\operatorname{Tr}\left(B^{3}\right) & =\operatorname{Tr}\left(\mu^{3}\right)+3 H \operatorname{Tr}\left(\mu^{2}\right)+n H^{3} \\
& =\operatorname{Tr}\left(\mu^{3}\right)+3 H|B|^{2}-2 n H^{3}
\end{aligned}
$$

Therefore, we have

$$
\left|\operatorname{Tr}\left(\mu^{3}\right)\right|=n(n-1)(n-2)\left|H^{3}\right|=\frac{n-2}{\sqrt{n(n-1)}}|\mu|^{3} .
$$

By Lemma 2.3, at any point $p \in M,\left(\mu_{i j}\right)=\operatorname{diag}\left\{v_{1}, \ldots, v_{1}, v_{2}\right\}$. Thus, we got at point $p$,

$$
h_{i j}=\operatorname{diag}\left\{v_{1}+H, \ldots, v_{1}+H, v_{2}+H\right\} .
$$

By Eq. (3.1) with $i=k=1$, we get

$$
\left(v_{1}+H\right)^{2}=\left((n-1)\left(v_{1}+H\right)+\left(v_{2}+H\right)\right)\left(v_{1}+H\right) ;
$$

and with $i=k=n$

$$
\left(v_{2}+H\right)^{2}=\left((n-1)\left(v_{1}+H\right)+\left(v_{2}+H\right)\right)\left(v_{2}+H\right) .
$$

Hence if $n=2$, we have $\left(v_{1}+H\right)\left(v_{2}+H\right)=0$, which means the sectional curvature is zero.

If $n \geq 3$, we conclude that $\left(v_{1}+H\right)=0$ which also implies that the sectional curvature of $M$ vanishes. By Hartman and Nirenberg's theorem, we know that $M$ is either $\mathbb{R}^{n}$ or $S^{1} \times \mathbb{R}^{n-1}$. Then we see that the total curvature of second case is not finite. Hence we complete the proof of Theorem 1.1.

\section{Proof of Theorem 1.2}

This section is devoted to the argument for our Theorem 1.2.

If $n=2$, by well-known result of Hartman and Nirenberg, any complete surfaces in $R^{3}$ with zero Gaussian curvature are either plane or cylinder. The later has infinity volume and non-zero constant mean curvature which cannot satisfy our finiteness assumption on the total integration of the square of the mean curvature. Therefore, this case follows. Thus in what follows, we assume $n \geq 3$ :

Proof of Theorem 1.2 (d) $\Rightarrow$ (a) is clear. 
(a) $\Rightarrow$ (b): $M$ is locally conformally flat; then $R_{i j, k}=R_{i k, j}$ since $R \equiv 0$. By the second Bianchi identity, one has

$$
\begin{aligned}
& R_{i j, l l} \\
= & R_{i l, j l} \\
= & R_{i l, l j}+R_{k l} R_{i j l}^{k}+R_{i k} R_{l j l}^{k} \\
= & \frac{2}{n-2}\left[R_{k l}\left(R_{k j} g_{i l}+R_{i l} g_{k j}-R_{k l} g_{i j}-R_{i j} g_{k l}\right)\right]+R_{i k} R_{k j} \\
= & \frac{2}{n-2}\left[R_{k i} R_{k j}+R_{j l} R_{i l}-\left(R_{k l}^{2}\right) g_{i j}-R_{i j} \cdot 0\right]+R_{i k} R_{k j} \\
= & \frac{2}{n-2}\left[2 R_{k i} R_{k j}-\left(R_{\alpha l}^{2}\right) g_{i j}\right]+R_{i k} R_{k j} .
\end{aligned}
$$

Therefore,

$$
R_{i j} \Delta R_{i j}=\frac{n+2}{n-2} R_{i j} R_{k i} R_{k j}
$$

It is well known that the following identity holds:

$$
R_{i j} \Delta R_{i j}+\left|\nabla R_{i j}\right|^{2}=\left|R_{i j}\right| \Delta\left|R_{i j}\right|+\left.|\nabla| R_{i j}\right|^{2} .
$$

Combining with previous calculation, one obtains

$$
\begin{aligned}
\left|R_{i j}\right| \Delta\left|R_{i j}\right| & =R_{i j} \Delta R_{i j}+\left|\nabla R_{i j}\right|^{2}-|\nabla| R_{i j}||^{2} \\
& =\frac{n+2}{n-2} R_{i j} R_{k i} R_{k j}+\left|\nabla R_{i j}\right|^{2}-|\nabla| R_{i j}||^{2} .
\end{aligned}
$$

Let $f$ be a cut-off function supported in a ball $B(o, R)$ with $o \in M$ such that $|\nabla f| \leq$ $\frac{C}{R},|\Delta f| \leq \frac{C}{R^{2}}$. Multiplying both sides of above identity by $f^{2}\left|R_{i j}\right|^{q}$ with $q>-1$, one reaches

$$
f^{2}\left|R_{i j}\right|^{q+1} \Delta\left|R_{i j}\right|=\frac{n+2}{n-2} f^{2} R_{i j} R_{\alpha i} R_{\alpha j}\left|R_{i j}\right|^{q}+\left(\left|\nabla R_{i j}\right|^{2}-\left.|\nabla| R_{i j}\right|^{2}\right) f^{2}\left|R_{i j}\right|^{q} .
$$

Integrate it and use integration by parts to get

$$
\begin{aligned}
& \int_{M} \nabla\left(f^{2}\left|R_{i j}\right|^{q+1}\right) \nabla\left|R_{i j}\right|+\frac{n+2}{n-2} \int_{M} f^{2} R_{i j} R_{k i} R_{k j}\left|R_{i j}\right|^{q} \\
& \quad+\int_{M}\left(\left|\nabla R_{i j}\right|^{2}-|\nabla| R_{i j}||^{2}\right) f^{2}\left|R_{i j}\right|^{q}=0,
\end{aligned}
$$

which can be rewritten as

$$
\begin{aligned}
& 0 \geq 2 \int_{M} f \nabla f\left|R_{i j}\right|^{q+1} \nabla\left|R_{i j}\right|+\left.(q+1) \int_{M} f^{2}\left|R_{i j}\right|^{q}|\nabla| R_{i j}\right|^{2} \\
& \quad+\frac{n+2}{n-2} \int_{M} f^{2} R_{i j} R_{k i} R_{k j}\left|R_{i j}\right|^{q}+\left.\frac{2}{n} \int_{M}|\nabla| R_{i j}\right|^{2} f^{2}\left|R_{i j}\right|^{q} .
\end{aligned}
$$

Here we have used the refined Kato's inequality:

$$
\left.|\nabla| R_{i j}\right|^{2} \leq \frac{n}{n+2}\left|\nabla R_{i j}\right|^{2} .
$$


This is well known since $R_{i j}$ is symmetric, trace free and $R_{i j, k}=R_{i k, j}$ for all $i, j$ and $k$.

Choose $q=\frac{n-4}{2}$. Notice that by Gauss formula,

$$
\begin{aligned}
\sum R_{i j}^{2} & =\left(n H h_{i j}-h_{i k} h_{k j}\right)^{2} \\
& =n^{4} H^{4}-2 n H \operatorname{tr}\left(B^{3}\right)+\sum_{i, j}\left(\sum_{k} h_{i k} h_{k j}\right)^{2} \\
& \leq 4 n^{4} H^{4} .
\end{aligned}
$$

It follows that $\int_{M}\left|R_{i j}\right|^{q+2}=\int_{M}\left|R_{i j}\right|^{n / 2}<\left(4 n^{4}\right)^{\frac{n}{4}} \int_{M}|H|^{n}<\left(4 n^{4}\right)^{\frac{n}{4}} \alpha^{n}<+\infty$. Thus

$$
\begin{aligned}
& -\frac{2}{q+2} \int_{M} \nabla(f \nabla f)\left|R_{i j}\right|^{q+2}+\frac{n+2}{n-2} \int_{M} f^{2} R_{i j} R_{k i} R_{k j}\left|R_{i j}\right|^{q} \\
& +\left.\left(q+1+\frac{2}{n}\right) \int_{M} f^{2}\left|R_{i j}\right|^{q}|\nabla| R_{i j}\right|^{2} \leq 0 .
\end{aligned}
$$

We can estimate the last term on the right-hand side as follows:

$$
\begin{aligned}
\left.\left|\int_{M} f^{2} R_{i j} R_{k i} R_{k j}\right| R_{i j}\right|^{q} \mid & \leq \int_{M} f^{2}\left|R_{j i}\right|^{q+3} \\
& =\int_{M}\left|R_{j i}\right|\left(f\left|R_{j i}\right|^{\frac{q}{2}+1}\right)^{2} \\
& \left.\leq \int_{M}\left(f\left|R_{j i}\right|^{\frac{q}{2}+1}\right)^{\frac{2 n}{n-2}} \mathrm{~d} v\right)^{\frac{n-2}{n}}\left(\int_{M}\left|R_{j i}\right|^{\frac{n}{2}} \mathrm{~d} v\right)^{\frac{2}{n}} \\
& \leq\left(\int_{M}\left|R_{j i}\right|^{\frac{n}{2}}\right)^{\frac{2}{n}} \cdot C_{s} \int_{M}\left|\nabla\left(f\left|R_{j i}\right|^{\frac{q}{2}+1}\right)\right|^{2} \\
& \leq 2\left(\int_{M}\left|R_{j i}\right|^{\frac{n}{2}}\right)^{\frac{2}{n}} \cdot C_{s}\left[\int_{M}|\nabla f|^{2}\left|R_{i j}\right|^{q+2}\right. \\
& \left.+\left.\left.\left(\frac{q}{2}+1\right)^{2} \int_{M} f^{2}\left|R_{i j}\right|\right|^{q}|\nabla| R_{i j}\right|^{2}\right]
\end{aligned}
$$

where $C_{s}$ is Sobolev constant given in (2.5). Combine this estimate and the Eq. (4.1) to get

$$
\begin{aligned}
& {\left.\left[\left(q+1+\frac{2}{n}\right)-2 \frac{n+2}{n-2}\left(\frac{n}{4}\right)^{2}\left(\int_{M}\left|R_{j i}\right|^{\frac{n}{2}}\right)^{\frac{2}{n}} \cdot C_{s}\right] \int_{M} f^{2}\left|R_{i j}\right|^{q}|\nabla| R_{i j}\right|^{2}} \\
& \leq 2 \frac{n+2}{n-2}\left(\int_{M}\left|R_{j i}\right|^{\frac{n}{2}}\right)^{\frac{2}{n}} \cdot C_{S} \int_{M}|\nabla f|^{2}\left|R_{i j}\right|^{q+2}+\frac{2}{q+2} \int_{M} \nabla(f \nabla f)\left|R_{i j}\right|^{q+2} .
\end{aligned}
$$


Therefore, if we choose

$$
\alpha=\frac{1}{C_{1}} \frac{\sqrt{\left(n^{2}-2 n+4\right)(n-2)^{3}}}{n \sqrt{\left(n^{2}-2 n+4\right)(n-2)^{3}}+\sqrt{2 n^{5}(n+2)(n-1)^{2}}},
$$

where $C_{1}$ is the constant given in Lemma 2.4, then we have

$$
\left(q+1+\frac{2}{n}\right)-2 \frac{n+2}{n-2}\left(\frac{n}{4}\right)^{2}\left(\int_{M}\left|R_{j i}\right|^{\frac{n}{2}}\right)^{\frac{2}{n}} \cdot C_{s}>0 .
$$

Thus let $R \rightarrow+\infty$ in the previous inequality; we obtain

$$
\nabla\left|R_{j i}\right|^{q / 2+1}=0 .
$$

Hence, $\left|R_{j i}\right|=$ const. But the facts that $\left|R_{j i}\right|^{\frac{n}{2}} \in L^{1}$ and $M$ has infinite volume imply that $\left|R_{j i}\right|=0$.

Then by Theorem $1.1, h_{i j}=0$. Therefore,

$$
|\nabla h|^{2}=n^{2}|\nabla H|^{2} .
$$

(b) $\Rightarrow$ (c): Observe that $\left(|\nabla h|^{2}-n^{2}|\nabla H|^{2}\right)|H|^{2} n^{2}=\frac{1}{2} \sum\left(h_{i j} h_{k l, t}-h_{i j, t} h_{k l}\right)^{2}$. Hence, the assumption (b) implies that $h_{i j} h_{k l, t}=h_{i j, t} h_{k l}$ for all $i, j, k, l$ and $t$.

Now Gauss equation implies $R_{i j}=n H h_{i j}-h_{i k} h_{k j}$. Thus we have

$$
\begin{aligned}
R_{i j, l} & =n H_{l} h_{i j}+n H h_{i j, l}-h_{i k, l} h_{k j}-h_{k j, l} h_{i k} \\
& =n H h_{i j, l}+n H h_{i l, j}-h_{i l, k} h_{k j}-h_{j l, k} h_{i k} \\
& =2 n H h_{i j, l}-h_{i l} h_{k j, k}-h_{j l} h_{i k, k} \\
& =2 n H h_{i l, j}-n H h_{i l, j}-n H h_{j l, i} \\
& =0 .
\end{aligned}
$$

Therefore, $\left|R_{i j}\right|$ is a constant, since $|d| R_{i j}|| \leq\left|\nabla R_{i j}\right|=0$. Hence, the integrability of $\left|R_{i j}\right|^{n / 2}$ implies that $R_{i j}=0$ which in turn implies that $h_{i j}=0$ by Theorem 1.1. Of course, the identity $n H \operatorname{tr}\left(B^{3}\right)=n^{4} H^{4}$ holds for $h_{i j}=0$.

(c) $\Rightarrow(\mathbf{d})$ : Note that

$$
\begin{aligned}
R_{i j}^{2} & =n^{4} H^{4}-2 n H \operatorname{tr}\left(B^{3}\right)+\sum\left(\sum_{k} h_{i k} h_{k j}\right)^{2} \\
& \leq 2 n^{4} H^{4}-2 n H \operatorname{tr}\left(B^{3}\right) \\
& =0 .
\end{aligned}
$$

Hence, $R_{i j}=0$. By Theorem 1.1, $M$ is flat.

\section{Proof Of Theorem 1.3}

In this section, we employ some methods due to Cao, Shen and Zhu to study hyper-surfaces with zero scalar curvature. We first have lower volume growth estimate as given in Lemma 2.6. Thus we can show that there exist bounded harmonic functions on such hyper-surfaces. We state it as a lemma. 
Lemma 5.1 Let $M$ be a complete and noncompact n-dimensional immersed hyper-surfaces in $\mathbb{R}^{n+1}$ satisfying $n\|H\|_{n} C_{1}<1$ where again $C_{1}$ is a constant given in Lemma 2.4. If $M$ has at least two ends, then $M$ admits a nonconstant bounded harmonic function with finite energy.

Proof The proof is analogy to the proof of Lemma 2 in [7]. We will provide the argument here for completeness of the paper. We first prove that for each compact set $K \subset M$, every noncompact component $F$ of $M \backslash K$ has infinite volume. Suppose $\operatorname{Vol}(F)$ were finite. By the fact that $\lim _{s \rightarrow \infty} s^{n\left(1-n\|H\|_{n} C_{1}\right)}=\infty$, there would exist a sufficiently large $s_{0}$ such that

$$
s_{0}^{n\left(1-n\|H\|_{n} C_{1}\right)}>\operatorname{Vol}(F) .
$$

Choosing a point $x_{0} \in F$ so that $r\left(x_{0}, \partial F\right)>s_{0}$ would lead to

$$
\operatorname{Vol}(F) \geq \operatorname{Vol}\left(B_{x_{0}}\left(s_{0}\right)\right) \geq s_{0}^{n\left(1-n C_{1}\|H\|_{n}\right)}
$$

which is a contradiction. Hence $\operatorname{Vol}(F)=\infty$.

Next let $M$ be covered by an exhaustion $\left\{D_{i}\right\}$, a collection of relatively compact submanifolds with boundary, for example, take $D_{i}=B(0, i) \cap M$ where $B(0, i)$ is the ball in $R^{n+1}$ with radial $i$ and center 0 . Let $M \backslash D_{i}=\cup_{j=1}^{s} F_{j}^{(i)}$ be the disjoint union of connected components with $s \geq 2$. Fix an $i_{0}$ and let $F_{1}^{\left(i_{0}\right)}$ and $F_{2}^{\left(i_{0}\right)}$ be any two ends; then each has infinite volume. For each $i \geq i_{0}$, let $u_{i}: D_{i} \rightarrow R$ be the minimizer of the energy functional $\frac{1}{2} \int_{D_{i}}\left|\mathrm{~d} u_{i}\right|^{2} \mathrm{~d} v$ among all functions $u$ such that $\left.u\right|_{\partial F_{1}^{(i)}}=1$ and $\left.u\right|_{\partial F_{k}^{(i)}}=0$ for each $k \geq 2$.

Then by the maximum principle for harmonic functions, $0 \leq u_{i} \leq 1$. For any $j<i$, we extend $u_{j}$ to $\bar{u}_{j}: D_{i} \rightarrow R$ continuously such that $\bar{u}_{j}=1$ or 0 on the complement $D_{i}-D_{j}$. Then $\bar{u}_{j}$ has the same boundary condition as $u_{i}$ on $\partial D_{i}$. Hence by the minimality of the energy $E\left(u_{i}\right)$ of $u_{i}$ over $D_{i}$, one has the following monotonicity:

$$
\int_{D_{i}}\left|\nabla u_{i}\right|^{2} \mathrm{~d} v \leq \int_{D_{i}}\left|\nabla \bar{u}_{j}\right|^{2} \mathrm{~d} v=\int_{D_{j}}\left|\nabla u_{j}\right|^{2} \mathrm{~d} v \text { for } i>j .
$$

Thus there exists a constant $c_{1}>0$ such that

$$
\int_{D_{i}}\left|\nabla u_{i}\right|^{2} \mathrm{~d} v \leq c_{1} \text { for } i>i_{0} .
$$

Therefore, we can find a harmonic function $u$ on $M$ such that

$$
\lim _{i \rightarrow \infty} u_{i}(x)=u(x), \quad \forall x \in M
$$

$0 \leq u \leq 1$ and $\int_{M}|\nabla u|^{2} \mathrm{~d} v \leq c_{1}$.

Since $n>2$, we substitute $f=u_{i}\left(1-u_{i}\right)$, in the inequality (2.5)

$$
\begin{aligned}
\left(\int_{D_{i}}\left(u_{i}\left(1-u_{i}\right)\right)^{\frac{2 n}{n-2}} \mathrm{~d} v\right)^{\frac{n-2}{n}} & \leq C_{s} \int_{D_{i}} 2\left|\nabla u_{i}\right|^{2}\left(1-u_{i}\right)^{2}+2 u_{i}^{2}\left|\nabla u_{i}\right|^{2} \mathrm{~d} v \\
& \leq 4 C_{s} \int_{D_{i}}\left|\nabla u_{i}\right|^{2} \leq 4 C_{s}^{2} c_{1} .
\end{aligned}
$$


Since $\operatorname{Vol}\left(D_{i}\right) \rightarrow \infty$, by letting $i \rightarrow \infty$, we find that if $u$ is a constant, then $u \equiv 0$ or $u \equiv 1$. If $u \equiv 1$, we choose $\phi=u_{i} \psi$ where

$$
\psi=\left\{\begin{array}{l}
1, \text { on } F_{2}^{\left(i_{0}\right)} \\
0, \text { on } F_{k}^{\left(i_{0}\right)}, k \neq 2
\end{array}\right.
$$

$|\nabla \psi| \leq c_{2}, 0 \leq \psi \leq 1$, and $|\nabla \psi|$ vanishes outside $D_{i_{0}}$, then inequality (2.5) implies that

$$
\left(\int_{D_{i}}\left(u_{i} \psi\right)^{\frac{2 n}{n-2}} \mathrm{~d} v\right)^{\frac{n-2}{n}} \leq C_{s} \int_{D_{i}} 2 \psi^{2}\left|\nabla u_{i}\right|^{2}+2 u_{i}^{2}|\nabla \psi|^{2} \mathrm{~d} v \leq c_{3}
$$

where the constant $c_{3}=2 C_{s}^{2} c_{1}+2 c_{s}^{2} c_{2}^{2} \cdot \operatorname{Vol}\left(D_{i_{0}}\right)$. It follows that

$$
\left(\int_{F_{2}^{\left(i_{0}\right)} \cap D_{i}} u_{i}^{\frac{2 n}{n-2}} \mathrm{~d} v\right)^{\frac{n-2}{n}} \leq c_{3}
$$

As $i \rightarrow \infty$, we find that $\operatorname{Vol}\left(F_{2}^{\left(i_{0}\right)}\right)^{\frac{n-2}{n}}=\left(\int_{F_{2}^{\left(i_{0}\right)}} u^{\frac{2 n}{n-2}} \mathrm{~d} v\right)^{\frac{n-2}{n}} \leq c_{3}$, a contradiction. Similarly, $u \equiv 0$ cannot happen by replacing $u$ and $u_{i}$ by $1-u$ and $1-u_{i}$, respectively, in same argument. Consequently, $u$ is not a constant. This completes the proof of Lemma 2.

Now we are ready to prove Theorem 1.3.

Proof of Theorem 1.3 We argue by contradiction. By the construction of Lemma 5.1, we know that if $M$ is of more than one end, then there exists a nontrivial bounded harmonic function $u(x)$ on $M$ which has finite total energy.

For such a harmonic function $u$, let $f(x)=|\nabla u|$. By Bochner formula, we obtain

$$
\frac{1}{2} \Delta f^{2}=|\operatorname{Hess} u|^{2}+\operatorname{Ric}(\nabla u, \nabla u)
$$

Next we prove the following inequality:

$$
\mid \text { Hess }\left.u\right|^{2} \geq\left(1+\frac{1}{n-1}\right)|\nabla f|^{2} \text {. }
$$

If $|\nabla u|=0$, the above inequality of course holds. If $|\nabla u| \neq 0$, for any $p \in M$ we choose a normal coordinate around $p$ such that $u_{i}(p)=0(i \geq 2)$ and $u_{1}(p)=|\nabla u|(p)$. Since $u$ is harmonic, we have 


$$
u_{11}=-\left(\sum_{i \neq 1} u_{i i}\right)
$$

Then at $p$,

$$
f_{j}=\frac{u_{i j} u_{i}}{|\nabla u|}=\frac{u_{1 j} u_{1}}{u_{1}}=u_{1 j} .
$$

Hence,

$$
|\nabla f|^{2}=u_{1 j}^{2}
$$

We can calculate that

$$
\begin{aligned}
\mid \text { Hess }\left.u\right|^{2}-|\nabla f|^{2} & =u_{i j}^{2}-u_{1 j}^{2} \\
& \geq \sum_{i \neq 1} u_{i 1}^{2}+\sum_{i \neq 1} u_{i i}^{2} \\
& \geq \sum_{i \neq 1} u_{i 1}^{2}+\frac{1}{n-1}\left(\sum_{i \neq 1} u_{i i}\right)^{2} \\
& =\sum_{i \neq 1} u_{i 1}^{2}+\frac{1}{n-1} u_{11}^{2} \\
& \geq \frac{1}{n-1} \sum_{i=1}^{n} u_{i 1}^{2} \\
& =\frac{1}{n-1}|\nabla f|^{2}
\end{aligned}
$$

since Hessian is symmetric. Therefore, we have proved (5.4).

Apply Leung's curvature estimate [12] with $k=0$ to get

$$
\begin{aligned}
\operatorname{Ric}_{\min } \geq & \frac{1}{n^{2}}\left\{2(n-1) n^{2} H^{2}-n(n-1)|B|^{2}\right. \\
& \left.-(n-2) n|H| \sqrt{(n-1)\left(n|B|^{2}-n^{2} H^{2}\right)}\right\} .
\end{aligned}
$$

Since the scalar curvature of the hyper-surface in our case is zero, by Lemma 2.1, we obtain

$$
\operatorname{Ric}_{\min } \geq-2(n-1)(n-2) H^{2} .
$$

With help of this estimate, Bochner formula takes the form,

$$
f \Delta f+2(n-1)(n-2) H^{2} f^{2} \geq \frac{1}{n-1}|\nabla f|^{2} .
$$

Now let $\varphi$ be a cut-off function such that

$$
\varphi(x)= \begin{cases}1, & \text { if } \quad x \in B_{p}(r), \\ 0, & \text { if } \quad x \in M \backslash B_{p}(2 r),\end{cases}
$$

and

$$
|\nabla \varphi| \leq \frac{C}{r} \text { with } C=2 .
$$

Multiplying $\varphi^{2}$ on both sides of the above inequality (5.5) and integrating by parts we can write it as 


$$
2(n-1)(n-2) \int_{M} H^{2} f^{2} \varphi^{2} \mathrm{~d} \sigma-2 \int_{M}\langle\nabla f, \nabla \varphi\rangle f \varphi \mathrm{d} \sigma \geq \frac{n}{n-1} \int_{M}|\nabla f|^{2} \varphi^{2} \mathrm{~d} \sigma .
$$

Using Schwartz inequality, for any positive number $\delta_{1}>0$, we have

$$
2(n-1)(n-2) \int_{M} H^{2} f^{2} \varphi^{2} \mathrm{~d} v+\frac{1}{\delta_{1}} \int_{M} f^{2}|\nabla \varphi|^{2} \mathrm{~d} v \geq\left(\frac{n}{n-1}-\delta_{1}\right) \int_{M}|\nabla f|^{2} \varphi^{2} \mathrm{~d} v .
$$

On the other hand, Sobolev inequality yields

$$
\int_{M}|\nabla(f \varphi)|^{2} \mathrm{~d} v \geq C_{S}^{-1}\left(\int_{M}(f \varphi)^{\frac{2 n}{n-2}} \mathrm{~d} v\right)^{\frac{n-2}{n}}
$$

Simple calculation, together with Schwartz inequality, yields

$$
\left(\delta_{2}+1\right) \int_{M}|\nabla f|^{2} \varphi^{2} \mathrm{~d} v \geq C_{s}^{-1}\left(\int_{M}(f \varphi)^{\frac{2 n}{n-2}} \mathrm{~d} v\right)^{\frac{n-2}{n}}-\left(1+\frac{1}{\delta_{2}}\right) \int_{M} f^{2}|\nabla \varphi|^{2} \mathrm{~d} v,
$$

where $\delta_{2}$ is a positive real number which will be chosen later. Combining (5.6) and (5.7), we have

$$
\begin{aligned}
2(n-1)(n-2) \int_{M} H^{2} f^{2} \varphi^{2} \mathrm{~d} v \geq & \frac{\left(\frac{n}{n-1}-\delta_{1}\right) C_{s}^{-1}}{\delta_{2}+1}\left(\int_{M}(f \varphi)^{\frac{2 n}{n-2}} \mathrm{~d} v\right)^{\frac{n-2}{n}} \\
& -\left(\frac{1}{\delta_{1}}+\frac{\frac{n}{n-1}-\delta_{1}}{\delta_{2}}\right) \int f^{2}|\nabla \varphi|^{2} \mathrm{~d} v .
\end{aligned}
$$

Now applying Hölder inequality to the left-hand side of the above inequality we can have

$$
\begin{aligned}
2(n-1)(n-2)\left(\int_{M}|H|^{n} \mathrm{~d} v\right)^{\frac{2}{n}}\left(\int_{M}(f \varphi)^{\frac{2 n}{n-2}} \mathrm{~d} v\right)^{\frac{n-2}{n}} \geq & \frac{\left(\frac{n}{n-1}-\delta_{1}\right) C_{s}^{-1}}{\delta_{2}+1}\left(\int_{M}(f \varphi)^{\frac{2 n}{n-2}} \mathrm{~d} v\right)^{\frac{n-2}{n}} \\
& -\left(\frac{1}{\delta_{1}}+\frac{\frac{n}{n-1}-\delta_{1}}{\delta_{2}}\right) \int_{M} f^{2}|\nabla \varphi|^{2} \mathrm{~d} v .
\end{aligned}
$$

Finally, we have

$$
\begin{aligned}
\left(\frac{1}{\delta_{1}}+\frac{\frac{n}{n-1}-\delta_{1}}{\delta_{2}}\right) \int_{M} f^{2}|\nabla \varphi|^{2} \mathrm{~d} v \geq & \left.\frac{\left(\frac{n}{n-1}-\delta_{1}\right) C_{s}^{-1}}{\delta_{2}+1}-2(n-1)(n-2)\|H\|_{n}^{2}\right) \\
& \times\left(\int_{M}(f \varphi)^{\frac{2 n}{n-2}} \mathrm{~d} v\right)^{\frac{n-2}{n}} .
\end{aligned}
$$

Recall that $C_{s}=\left(\frac{C_{1}}{1-n\|H\|_{n} C_{1}} \frac{2(n-1)}{n-2}\right)^{2}$; thus if we choose 


$$
C_{2}=\frac{1}{C_{1}}\left(\frac{\sqrt{n(n-2)}}{2 \sqrt{2}(n-1)^{2}+n \sqrt{n(n-2)}}\right),
$$

then it is easy to see that if $\|H\|_{n}<C_{2}$, then $\|H\|_{n}^{2} C_{s}<\frac{n}{2(n-1)^{2}(n-2)}$. Thus we can choose $\delta_{1}>0$ and $\delta_{2}>0$ small enough such that

$$
\left(\frac{\left(\frac{n}{n-1}-\delta_{1}\right) C_{s}^{-1}}{\delta_{2}+1}-2(n-1)(n-2)\|H\|_{n}^{2}\right) \geq \epsilon>0 .
$$

Then we have

$$
\left(\frac{1}{\delta_{1}}+\frac{\frac{n}{n-1}-\delta_{1}}{\delta_{2}}\right) \int_{M} f^{2}|\nabla \varphi|^{2} \mathrm{~d} v \geq \epsilon\left(\int_{M}(f \varphi)^{\frac{2 n}{n-2}} \mathrm{~d} v\right)^{\frac{n-2}{n}} .
$$

Letting $r \rightarrow \infty$ we will have

$$
\int_{M} f^{\frac{2 n}{n-2}} \mathrm{~d} v \leq 0
$$

which implies that $f \equiv 0$ and, therefore, $u$ is a constant function. The contradiction here shows that $M$ has at most one end.

Remark 1. The zero scalar curvature equation for a graph has been derived by Reilly [16]. It is given by: $\sum_{i, j} \frac{\partial}{\partial x_{i}}\left(\frac{1}{W} T_{j}^{i} f_{j}\right)=0$, where the function $f$ is a function such that $x_{n+1}=$ $f\left(x_{1}, x_{2}, \ldots, x_{n+1}\right)$, and $W=1+|D f|^{2}, T_{j}^{i}=\sum_{k} \frac{\partial}{\partial x_{k}}\left(f_{k} / W\right) \delta_{i j}-\frac{f_{i j}}{W}+\sum_{k} \frac{f_{j k} f_{k} f_{i}}{W^{3}}$. It also can be written as follows: $\left(1+\sum f_{i}^{2}\right)\left[\left(\sum f_{i i}\right)^{2}-\sum f_{j k}^{2}\right]+2 \sum f_{j k} f_{j i} f_{k} f_{i}-$ $2 \sum f_{i i} f_{j k} f_{j} f_{k}=0$, where the sums are taken over all the repeated indices, respectively, and

$$
f_{i}=\frac{\partial}{\partial x_{i}} f, f_{j k}=\frac{\partial^{2}}{\partial x_{j} \partial x_{k}} f .
$$

Therefore, we may express the zero scalar curvature equation as the following:

$$
\begin{aligned}
0=(1 & \left.+|\nabla f|^{2}\right)\left[(\Delta f)^{2}-|\operatorname{Hess}(f)|^{2}\right] \\
& +\left.2|\nabla f|^{2}|\nabla| \nabla f\right|^{2}-2 \Delta f \cdot \operatorname{Hess}(f)(\nabla f, \nabla f) .
\end{aligned}
$$

2. Our Theorem 1.2 says that under suitable condition on $\operatorname{div}\left(\frac{\nabla f}{W}\right)$, Eq. (5.9) has only linear solutions.

Acknowledgments The project started several years ago when the second author visited Institute of Mathematical Science at Nanjing University. He would like to thank them for hospitality and financial support by Siyuan foundation. Second author is also supported by NUS research grant R-000-127-112.

Open Access This article is distributed under the terms of the Creative Commons Attribution License which permits any use, distribution, and reproduction in any medium, provided the original author(s) and the source are credited.

\section{References}

1. Alencar, H., do Carmo, M. (BR-IMPA), Colares, A. G. (BR-FCR).: Stable hypersurfaces with constant scalar curvature. Math. Z. 213(1), 117-131 (1993) 
2. Alencar, Hilrio, do Carmo, Manfredo, Elbert, Maria Fernanda: Stability of hypersurfaces with vanishing $r$-mean curvatures in Euclidean spaces. J. Reine Angew. Math. 554, 201-216 (2003)

3. Calabi, E.: Examples of Bernstein problems for some nonlinear equations. Proc. Symp. Pure Appl. Math. 15, 223-230 (1968)

4. do Carmo, Manfredo P., Zhou, Detang: Bernstein-type theorems in hypersurfaces with constant mean curvature. An. Acad. Brasil. Cinc 72(3), 301-310 (2000)

5. Cheung, Leung-fu, Zhou, Detang: Stable constant mean curvature hypersurfaces in $\mathbb{R}^{n+1}$ and $\mathbb{H}^{n+1}(-1)$. Bull. Braz. Math. Soc. (N.S.) 36(1), 99-114 (2005)

6. Castro, Ildefonso, Montealegre, Cristina R.: Lagrangian submanifolds with zero scalar curvature in complex Euclidean space. Geom. Dedicata 86(1-3), 179-183 (2001)

7. Cao, H.-D., Shen, Y., Zhu, S.: The structure of stable minimal hypersurfaces in $R^{n+1}$. Math. Res. Lett. 4(4), 637-644 (1997)

8. Fleming, W.H.: On the oriented Plateau problem. Circolo Mat. Palermo II, 1-22 (1962)

9. Hartman, P., Nirenberg, L.: On spherical image maps whose Jacobians do not change sign. Am. J. Math. 81, 901-920 (1959)

10. Hounie, Jorge, Leite, Maria Luiza: Two-ended hypersurfaces with zero scalar curvature. Indiana Univ. Math. J. 48(3), 867-882 (1999)

11. Hoffman, D., Spruck, J.: Sobolev and isoperimetric inequalities for Riemannian submanifolds. Comm. Pure Appl. Math. 27, 715-727 (1975)

12. Leung, P.F.: An estimate on the Ricci curvature of a submanifold and some applications. Proc. AMS 114(4), 1051-1061 (1992)

13. Hounie, J., Leite, M.L.: Two ends hypersurfaces with zero scalar curvature. Indiana Univ. Math. J. 74(3), 1129-1145 (1998)

14. Michael, J., Simon, L.: Sobolev and mean-value inequalities on generalized submanifolds of $R^{n}$. Comm. Pure Appl. Math. 26, 361-379 (1973)

15. Okumura, Masafumi: Hypersurfaces and a pinching problem on the second fundamental tensor. Am. J. Math. 96, 207-213 (1974)

16. Reilly, R.: On the Hessian of a function and the curvatures of its graph. Mich. Math. J. 20, 373-383 (1973)

17. Sato, Jocelino: Stability of $\mathrm{O}(p+1) \times \mathrm{O}(p+1)$-invariant hypersurfaces with zero scalar curvature in Euclidean space. Ann. Global Anal. Geom 22(2), 135-153 (2002)

18. Shen, Y.-B., Zhu, X.-H.: On stable minimal hypersurfaces in $R^{n+1}$. Am. J. Math. 120, 103-116 (1998)

19. Shoen, R., Simon, L., Yau, S.-Y.: Curvature estimates for minimal hypersurfaces. Acta Math. 134, 275288 (1975)

20. Yun, Gabjin: Total scalar curvature and rigidity of minimal hypersurfaces in Euclidean space. Mathematika 48(1-2), 247-251 (2001) 